OPEN ACCESS

Edited by:

Wolfram Weckwerth,

University of Vienna, Austria

Reviewed by:

Christian Fleck

ETH Zürich, Switzerland

*Correspondence: Xiaohan Yang yangx@ornl.gov

Specialty section:

This article was submitted to Plant Systems and Synthetic Biology,

a section of the journal

Frontiers in Plant Science

Received: 18 December 2019 Accepted: 18 March 2020 Published: 09 April 2020

Citation:

Yang X, Cushman JC, Borland AM and Liu Q (2020) Editorial: Systems Biology and Synthetic Biology in Relation to Drought Tolerance or Avoidance in Plants.

Front. Plant Sci. 11:394.

doi: $10.3389 /$ fpls.2020.00394

\section{Editorial: Systems Biology and Synthetic Biology in Relation to Drought Tolerance or Avoidance in Plants}

\author{
Xiaohan Yang ${ }^{1,2 \star}$, John C. Cushman ${ }^{3}$, Anne M. Borland ${ }^{4}$ and Qingchang Liu ${ }^{5}$ \\ ${ }^{1}$ Biosciences Division, Oak Ridge National Laboratory, Oak Ridge, TN, United States, ${ }^{2}$ Oak Ridge National Laboratory, The \\ Center for Bioenergy Innovation, Oak Ridge, TN, United States, ${ }^{3}$ Department of Biochemistry and Molecular Biology, \\ University of Nevada, Reno, NV, United States, ${ }^{4}$ School of Natural and Environmental Science, Newcastle University, \\ Newcastle upon Tyne, United Kingdom, ${ }^{5}$ Key Laboratory of Sweet Potato Biology and Biotechnology, Ministry of Agriculture, \\ Beijing Key Laboratory of Crop Genetic Improvement, Laboratory of Crop Heterosis and Utilization, Ministry of Education, \\ College of Agronomy \& Biotechnology, China Agricultural University, Beijing, China
}

Keywords: genomics, synthetic biology, systems biology, drought tolerance, drought avoidance, crassulacean acid metabolism

Editorial on the Research Topic

Systems Biology and Synthetic Biology in Relation to Drought Tolerance or Avoidance in Plants

Drought stress has been a long-time limitation to crop production that is being exacerbated by climate change and associated reductions in the availability of blue water resources for agriculture. Most existing food and industrial crops are susceptible to drought stress, which can cause a significant loss in crop yield. Therefore, our ability to develop more climate-resilient crops that are more heat and drought tolerant will become increasingly important in the near future. In nature, plants have evolved two important mechanisms to overcome the effects of drought stress: (1) drought avoidance, which enables plants to maintain relatively high tissue water content in water-limited environments by minimizing water loss and optimizing water uptake, and (2) drought tolerance, which enables plants to endure low tissue water content by maintaining cell turgor (resulting from osmotic adjustment and cellular elasticity) and increasing protoplasmic resistance (Basu et al., 2016). With more and more genomics resources available for diverse plant lineages showing contrasting strategies and variation in drought avoidance or tolerance (Yin et al., 2014; Abraham et al., 2016; Yang et al., 2017; Chen et al., 2018), systems biology, which features genome-scale analysis of molecules and their interactions (Westerhoff and Palsson, 2004), is becoming a popular approach to link genes to drought-avoidance or drought-tolerance traits. Our knowledge about the genes associated with drought stress responses generated by systems biology research can inform the construction of libraries of biological parts for synthetic biology, which aims to design or re-design biological processes (Cook et al., 2014). Synthetic biology has great potential for creating genetically-modified plants with enhanced drought avoidance or tolerance (Borland et al., 2014; De Paoli et al., 2014; Llorente et al., 2018). This Research Topic features three articles on the theme of systems biology of crassulacean acid metabolism (CAM) as a model strategy for plant adaptation to water-limited conditions and four articles related to genetic improvement of plant drought avoidance or tolerance using synthetic biology and genetic engineering approaches. 


\section{IDENTIFICATION OF CAM-RELATED GENES USING SYSTEMS BIOLOGY APPROACH}

CAM photosynthesis enables drought avoidance and high water-use efficiency (WUE) in plants through an inverted day/night pattern of stomatal movement relative to $\mathrm{C}_{3}$ or $\mathrm{C}_{4}$ photosynthesis plants, with stomata closed during daytime to reduce water loss via evapotranspiration (Borland et al., 2009; Yang et al., 2015). In this Research Topic, Moseley, Tuskan et al., compared diel gene expression patterns between one obligate CAM species Kalanchoë fedtschenkoi and two $\mathrm{C}_{3}$ photosynthesis species (Arabidopsis thaliana and Solanum lycopersicum). They identified 16 ortholog groups (OGs) containing stomata-related genes showing rescheduled (dawn vs. dusk) gene expression in the CAM species in comparison with the two $\mathrm{C}_{3}$ species. Furthermore, they performed evolutionary genomics analysis of these 16 OGs and highlighted several genes, such as serine/threonine-protein kinase nak1 and Catalase 2, as candidates regulating stomatal movement in CAM plants via abscisic acid (ABA) signaling and hydrogen peroxide $\left(\mathrm{H}_{2} \mathrm{O}_{2}\right)$ signaling, respectively. Also, in this Research Topic, Heyduk et al. performed comparative analysis of time-course gene expression between two closely-related species in the genus Erycina, E. pusilla and E. crista-galli, which are CAM and $\mathrm{C}_{3}$ photosynthesis plants, respectively. Their analysis revealed differential expression networks of genes involved in light sensing and $\mathrm{ABA}$ signaling between the $\mathrm{C}_{3}$ and CAM Erycina species. CAM physiology is likely controlled by the circadian clock (Boxall et al., 2017; Yang et al., 2017). In this Research Topic, Moseley, Mewalal et al. performed genome-wide prediction of rhythmic gene sets in the CAM species $K$. fedtschenkoi and $\mathrm{C}_{3}$ photosynthesis species $A$. thaliana through analysis of time-course gene expression data and identified CAM-related rhythmic genes, which displayed phase shifts between these two species. Recently, knock-down and knock-out mutant lines were generated for some CAM-related genes using RNA interference (RNAi) (Boxall et al., 2017, 2020) or genome-editing mediated by clustered regularly interspaced short palindromic repeats (CRISPR) and CRISPR-associated protein 9 (Cas9) (Liu et al., 2019). Multi-scale modeling of CAM systems at the molecular, cellular, and leaf level is needed to gain a deep understanding of gene and metabolic networks associated with CAM physiology (Liu et al., 2018). In the future, multi-omics (i.e., genomics, transcriptomics, metabolomics, proteomics) data will need to be generated for CAM plant species and their mutant lines for constructing gene and metabolic networks relevant to CAM.

\section{GENETIC IMPROVEMENT OF PLANT DROUGHT RESISTANCE USING SYNTHETIC BIOLOGY AND GENETIC ENGINEERING APPROACHES}

Engineering of CAM-related genes in $\mathrm{C}_{3}$ plants has great potential for genetic improvement of water-use efficiency and drought resistance (Borland et al., 2014; Yang et al., 2015).
Comparative analysis of leaf metabolic networks between CAM and $\mathrm{C}_{3}$ plants indicates that CAM-engineering in $\mathrm{C}_{3}$ crops could result in a major increase in water-use efficiency without substantial negative impact on yield (Shameer et al., 2018). CAM-engineering is likely to prove a challenging task, requiring a synthetic biology approach for precise control of temporal and spatial expression of multiple gene modules responsible for carboxylation, decarboxylation, stomatal movement, and leaf anatomy. In this Research Topic, Lim et al. showed exciting progress in transferring individual CAM carboxylation and decarboxylation genes from the facultative CAM species Mesembryanthemum crystallinum (ice plant) to the $\mathrm{C}_{3}$ species A. thaliana. Over-expressing each individual gene of the $M$. crystallinum carboxylation module (containing 6 genes) and decarboxylation module (containing 7 genes), except for three genes (McNADP-MDH1, McPPDK-RP, and McPEPCK), under the control of the constitutive CaMV $35 \mathrm{~S}$ promoter, increased plant size (in term of rosette diameter, leaf area, and leaf fresh weight) in the transgenic A. thaliana plants. Overexpression of most carboxylation genes increased stomatal conductance and acid accumulation while over-expression of the decarboxylating malic enzymes reduced stomatal conductance and acid accumulation. This study is an important milestone in CAM-engineering. Future research will be needed to test the coordinated over-expression of combinations of these carboxylation and decarboxylation genes in the same temporal and spatial manner as displayed by CAM species.

In general, drought stress experienced by crop plants is seasonal. Therefore, engineering drought-inducible CAM (or CAM-on-demand) systems would be ideal. In nature, CAM can be induced by drought stress in facultative CAM plants, which perform $\mathrm{C}_{3}$ photosynthesis under well-watered conditions (Winter, 2019; Yang et al., 2019a). In this Research Topic, Amin et al. proposed a strategy for engineering of CAMon-demand systems based on the engineering of droughtresponsive transcription factors (TFs) in multiple gene families (e.g., AP2/ERF, MYB, WRKY, NAC, NF-Y, bZIP) from the facultative CAM plant M. crystallinum and obligate CAM plant $K$. fedtschenkoi. The study showed that overexpression of a drought-responsive NAC family gene from K. fedtschenkoi (KfNAC83) in A. thaliana enhanced resistance to water-deficit stress and increased WUE. In the future, establishing the regulatory relationship between the drought-responsive TFs and CAM pathway genes will be necessary to establish CAM-ondemand systems.

Drought-responsive genes in $\mathrm{C}_{3}$ or $\mathrm{C}_{4}$ photosynthesis plant species are a rich source of target genes for genetic engineering to improve drought tolerance (Umezawa et al., 2006; Kamthan et al., 2016). In this Research Topic, Chen et al. increased the drought tolerance in rice through over-expression of a droughtinducible rice gene OsNAR2.1 encoding a nitrate transporter partner protein. Also, in this Research Topic, Lian et al. demonstrated that ectopic expression of a Populus trichocarpa gene (PtNF-YA9), which encodes a NUCLEAR FACTOR Y transcription factor, enhanced drought tolerance in the vegetative growth stage in Arabidopsis. A key focus for future research will be to test if overexpression of PtNF-YA9 can increase 
biomass production in poplar, an important $\mathrm{C}_{3}$ bioenergy crop, under drought conditions. Besides OsNAR2.1 and PtNF-YA9, many other genes are also responsible for drought resistance (Singh et al., 2019). In the future, genetic circuits (e.g., toggle switches, feedback loops, Boolean logic gates) (Kassaw et al., 2018) combining CAM genes and drought responsive TFs through iterative design-build-test-learn cycles will be required for drought avoidance and drought tolerance, respectively. For example, Boolean logic gates, which utilize Boolean algebra to convert multiple input signals into truth values of 1 if true or 0 if false (Andres et al., 2019), can be used to control the expression of genes related to drought tolerance through integrating droughtinduced positive and negative transcriptional regulators. Also, synthetic oscillator comprising positive and negative feedback loops (Andres et al., 2019) and circadian clock-regulated toggle switch (Schmal et al., 2013) need to be created for controlling the temporal (i.e., day vs. night) expression of genes related to CAM and stomatal movement in the $C_{3}$ plant species.

\section{CONCLUDING REMARKS}

This Research Topic has identified important candidate genes underpinning drought avoidance and tolerance through systems biology research and has also demonstrated the potential of synthetic biology and genetic engineering for increasing drought resistance in plants. With genomics research shifting from genome-reading to genome-editing and rewriting (Yang et al., 2019b), the knowledge generated in this Research Topic will facilitate future efforts in designing climate-resilient crops for reducing yield losses and expanding the production of food and bioenergy crops to marginal lands.

\section{REFERENCES}

Abraham, P. E., Yin, H., Borland, A. M., Weighill, D., Lim, S. D., De Paoli, H. C., et al. (2016). Transcript, protein and metabolite temporal dynamics in the CAM plant Agave. Nat. Plants 2:16178. doi: 10.1038/nplants.2016.178

Andres, J., Blomeier, T., and Zurbriggen, M. D. (2019). Synthetic switches and regulatory circuits in plants. Plant Physiol. 179, 862-884. doi: $10.1104 /$ pp. 18.01362

Basu, S., Ramegowda, V., Kumar, A., and Pereira, A. (2016). Plant adaptation to drought stress. F1000Res. 5:F1000 Faculty Rev-1554. doi: 10.12688/f1000research.7678.1

Borland, A. M., Griffiths, H., Hartwell, J., and Smith, J. A. C. (2009). Exploiting the potential of plants with crassulacean acid metabolism for bioenergy production on marginal lands. J. Exp. Bot. 60, 2879-2896. doi: 10.1093/jxb/erp118

Borland, A. M., Hartwell, J., Weston, D. J., Schlauch, K. A., Tschaplinski, T. J., Tuskan, G. A., et al. (2014). Engineering crassulacean acid metabolism to improve water-use efficiency. Trends Plant Sci. 19, 327-338. doi: 10.1016/j.tplants.2014.01.006

Boxall, S. F., Dever, L. V., Knerová, J., Gould, P. D., and Hartwell, J. (2017). Phosphorylation of phosphoenolpyruvate carboxylase is essential for maximal and sustained dark $\mathrm{CO}_{2}$ fixation and core circadian clock operation in the obligate crassulacean acid metabolism species Kalanchoë fedtschenkoi. Plant Cell 29, 2519-2536. doi: 10.1105/tpc.17.00301

Boxall, S. F., Kadu, N., Dever, L. V., Knerova, J., Waller, J. L., Gould, P. D., et al. (2020). Kalanchoë PPC1 is essential for crassulacean acid metabolism and the regulation of core circadian clock and guard cell signaling genes. Plant Cell. doi: $10.1105 /$ tpc. 19.00481

\section{AUTHOR CONTRIBUTIONS}

All authors listed have made a substantial, direct and intellectual contribution to the work, and approved it for publication.

\section{FUNDING}

The writing of this manuscript was supported by the Department of Energy (DOE), Office of Science, Genomic Science Program under Award Number DE-SC0008834 and the Center for Bioenergy Innovation (CBI), a U.S. Department of Energy (DOE) Bioenergy Research Center supported by the Office of Science, Office of Biological and Environmental Research (OBER).

\section{ACKNOWLEDGMENTS}

This manuscript has been authored by UT-Battelle, LLC under Contract No. DE-AC05-00OR22725 with the U.S. Department of Energy. The United States Government retains and the publisher, by accepting the article for publication, acknowledges that the United States Government retains a non-exclusive, paidup, irrevocable, world-wide license to publish, or reproduce the published form of this manuscript, or allow others to do so, for United States Government purposes. The Department of Energy will provide public access to these results of federally sponsored research in accordance with the DOE Public Access Plan (http://energy.gov/downloads/doe-public-access-plan). Oak Ridge National Laboratory is managed by UT-Battelle, LLC for the U.S. Department of Energy under Contract Number DE-AC05-00OR22725.

Chen, F., Dong, W., Zhang, J., Guo, X., Chen, J., Wang, Z., et al. (2018). The sequenced angiosperm genomes and genome databases. Front. Plant Sci. 9:418. doi: 10.3389/fpls.2018.0 0418

Cook, C., Martin, L., and Bastow, R. (2014). Opportunities in plant synthetic biology. J. Exp. Bot. 65, 1921-1926. doi: 10.1093/jxb/eru013

De Paoli, H. C., Borland, A. M., Tuskan, G. A., Cushman, J. C., and Yang, X. (2014). Synthetic biology as it relates to CAM photosynthesis: challenges and opportunities. J. Exp. Bot. 65, 3381-3393. doi: 10.1093/jxb/eru038

Kamthan, A., Chaudhuri, A., Kamthan, M., and Datta, A. (2016). Genetically modified (GM) crops: milestones and new advances in crop improvement. Theor. Appl. Genet. 129, 1639-1655. doi: 10.1007/s00122-016-2747-6

Kassaw, T. K., Donayre-Torres, A. J., Antunes, M. S., Morey, K. J., and Medford, J. I. (2018). Engineering synthetic regulatory circuits in plants. Plant Sci. 273, 13-22. doi: 10.1016/j.plantsci.2018.04.005

Liu, D., Chen, M., Mendoza, B., Cheng, H., Hu, R., Li, L., et al. (2019). CRISPR/Cas9-mediated targeted mutagenesis for functional genomics research of crassulacean acid metabolism plants. J. Exp. Bot. 70, 6621-6629. doi: $10.1093 / \mathrm{jxb} / \mathrm{erz} 415$

Liu, D., Palla, K. J., Hu, R., Moseley, R. C., Mendoza, C., Chen, M., et al. (2018). Perspectives on the basic and applied aspects of crassulacean acid metabolism (CAM) research. Plant Sci. 274, 394-401. doi: 10.1016/j.plantsci.2018.06.012

Llorente, B., Williams, T. C., and Goold, H. D. (2018). The multiplanetary future of plant synthetic biology. Genes 9:348. doi: 10.3390/genes9070348

Schmal, C., Reimann, P., and Staiger, D. (2013). A circadian clock-regulated toggle switch explains AtGRP7 and AtGRP8 oscillations in Arabidopsis thaliana. PLoS Comput. Biol. 9:e1002986. doi: 10.1371/journal.pcbi.1002986 
Shameer, S., Baghalian, K., Cheung, C. Y. M., Ratcliffe, R. G., and Sweetlove, L. J. (2018). Computational analysis of the productivity potential of CAM. Nat. Plants 4, 165-171. doi: 10.1038/s41477-018-0112-2

Singh, P. K., Srivastava, D., Tiwari, P., Tiwari, M., Verma, G., and Chakrabarty, D. (2019) "Drought tolerance in plants: molecular mechanism and regulation of signaling molecules," in Plant Signaling Molecules, eds M. I. R. Khan, P. S. Reddy, A. Ferrante, and N. A. Khan (Cambridge: Woodhead Publishing), 105-123. doi: 10.1016/B978-0-12-816451-8.00006-X

Umezawa, T., Fujita, M., Fujita, Y., Yamaguchi-Shinozaki, K., and Shinozaki, K. (2006). Engineering drought tolerance in plants: discovering and tailoring genes to unlock the future. Curr. Opin. Biotechnol. 17, 113-122. doi: 10.1016/j.copbio.2006.02.002

Westerhoff, H. V., and Palsson, B. O. (2004). The evolution of molecular biology into systems biology. Nat. Biotechnol. 22, 1249-1252. doi: 10.1038/nbt1020

Winter, K. (2019). Ecophysiology of constitutive and facultative CAM photosynthesis. J. Exp. Bot. 70, 6495-6508. doi: 10.1093/jxb/erz002

Yang, X., Cushman, J. C., Borland, A. M., Edwards, E. J., Wullschleger, S. D., Tuskan, G. A., et al. (2015). A roadmap for research on crassulacean acid metabolism (CAM) to enhance sustainable food and bioenergy production in a hotter, drier world. New Phytol. 207, 491-504. doi: 10.1111/ nph.13393

Yang, X., Hu, R., Yin, H., Jenkins, J., Shu, S., Tang, H., et al. (2017). The Kalanchoë genome provides insights into convergent evolution and building blocks of crassulacean acid metabolism. Nat. Commun. 8:1899. doi: 10.1038/s41467-017-01491-7

Yang, X., Liu, D., Tschaplinski, T. J., and Tuskan, G. A. (2019a). Comparative genomics can provide new insights into the evolutionary mechanisms and gene function in CAM plants. J. Exp. Bot. 70, 6539-6547. doi: 10.1093/jxb/erz408

Yang, X., Qi, L. S., Jaramillo, A., and Cheng, Z.-M. (2019b). Biodesign research to advance the principles and applications of biosystems design. Biodesign Res. 2019:9680853. doi: 10.34133/2019/9680853

Yin, H., Chen, C. J., Yang, J., Weston, D. J., Chen, J.-G., Muchero, W., et al. (2014). Functional genomics of drought tolerance in bioenergy crops. Crit. Rev. Plant Sci. 33, 205-224. doi: 10.1080/07352689.2014.870417

Conflict of Interest: The authors declare that the research was conducted in the absence of any commercial or financial relationships that could be construed as a potential conflict of interest.

Copyright (c) 2020 Yang, Cushman, Borland and Liu. This is an open-access article distributed under the terms of the Creative Commons Attribution License (CC BY).

The use, distribution or reproduction in other forums is permitted, provided the original author(s) and the copyright owner(s) are credited and that the original publication in this journal is cited, in accordance with accepted academic practice. No use, distribution or reproduction is permitted which does not comply with these terms. 